\title{
S Research Square \\ Immunohistochemical detection of preproenkephalin in the mouse brain
}

\section{Liliana Minichiello ( $\boldsymbol{Q}$ liliana.minichiello@pharm.ox.ac.uk)}

(1)Centre for Neuroregeneration, University of Edinburgh, EH16 4SB, Edinburgh, UK. (2)European Molecular Biology Laboratory, Mouse Biology Unit, 00015-Monterotondo, Rome, Italy. (3)Department of Pharmacology, University of Oxford, OX1 3QT, Oxford, UK

\section{Dario Besusso}

(1)Centre for Neuroregeneration, University of Edinburgh, EH16 4SB, Edinburgh, UK. (2)European Molecular Biology Laboratory, Mouse Biology Unit, 00015-Monterotondo, Rome, Italy.

\section{Mirjam Geibel}

(1)Centre for Neuroregeneration, University of Edinburgh, EH16 4SB, Edinburgh, UK. (2)European Molecular Biology Laboratory, Mouse Biology Unit, 00015-Monterotondo, Rome, Italy. (3)Department of Pharmacology, University of Oxford, OX1 3QT, Oxford, UK

\section{Method Article}

Keywords: Preproenkephalin, penk1, immunohistochemistry, fluorescent immunostaining, striatum, mouse brain, Huntington's disease.

Posted Date: June 18th, 2013

DOI: https://doi.org/10.1038/protex.2013.059

License: (a) This work is licensed under a Creative Commons Attribution 4.0 International License. Read Full License 


\section{Abstract}

Huntington's disease $\backslash(\mathrm{HD})$ is a genetic neurological disorder characterized by early degeneration of enkephalin-expressing striatal neurons, also known as Medium-sized Spiny Neurons $\backslash(M S N s)$. Therefore, accurate immunodetection of enkephalin expressing cells is of wide interest for the scientific community. Here, we describe an immunohistochemical procedure for the detection of the unprocessed precursor of the opioid peptide met-enkephalin, namely the preproenkephalin \(Penk1), in mouse brain cryosections. The specificity of the immunolabeling has been confirmed by crossing a reporter mouse line whereby EYFP expression is obtained upon Cre-mediated recombination in animals carrying Cre recombinase under the control of the preproenkephalin promoter.

\section{Introduction}

Huntington disease is an autosomal dominant and progressive neurodegenerative disorder characterized by a severe deficit in motor function, cognitive and psychiatric changes. ${ }^{1}$. Pathogenesis is caused by an expansion of a CAG trinucleotide repeat at the $\mathrm{N}$-terminus region of the huntingtin gene $\backslash(\mathrm{mhtt})^{2}$. In the earliest stages, the neurodegeneration is highly selective for striatal GABAergic MSNs projecting to the external pars of the globus pallidum ${ }^{3}$. These cells belong to the indirect pathway of the basal ganglia circuitry and express the neuropeptide enkephalin ${ }^{4}$. Neuronal degeneration appears to be anticipated by reduction in enkephalin expression ${ }^{5}$. For these reasons accurate and specific immunodetection of enkephalin-expressing cells is a fundamental tool for the research in the field allowing quantification of peptide level and cell type-specific measurements. Here, we describe a protocol for fluorescent detection of the preproenkephalin \(Penk1) peptide in mouse brain cryosections. Penk1 represents the precursor of the active form of the opioid peptide met-enkephalin which is obtained upon proteolitic cleavage ${ }^{6}$. Enkephalins represent endogenous ligands for the $\delta$-opioid receptor and are involved in the process of nociception ${ }^{7}$. In our experience, fundamental to the success of the procedure is the fixation of the tissue, and the use of freshly cut cryosections for the immunostaining. Prolonged exposure to crosslinking fixative has the tendency to mask the enkephalin epitope compromising its detection.

\section{Reagents}

-Pentobarbital sodium salt \(Sigma-Aldrich, P3761) -Tris Buffer Solution \(TBS): $50 \mathrm{mM}$ Tris pH 7.4; 150 $\mathrm{mM} \mathrm{NaCl}$-Washing Solution \(WS): $0.1 \%$ Tween 20 in TBS -Crosslinking Solution \(CS): 4\% PFA in TBS Cryo-Preservation Buffer $\backslash(\mathrm{CPB})$ : $30 \%$ sucrose in TBS -Quenching Solution $\backslash(\mathrm{QS})$ : $0.1 \mathrm{M}$ ammonium chloride, $0.1 \mathrm{M}$ glicine in TBS -Blocking Buffer $\backslash(\mathrm{BB})$ : 4\% NGS, 0.3\% Carrageen, 0.5\% TritonX-100 in TBS Antibody Incubation Buffer \(AIB): 1\% NGS, 0.3\% Carrageen, 0.1\% TritonX-100 in TBS -Sodium Citrate Buffer \(SCB): 10 mM Sodium Citrate, 0.05\% Tween 20, pH 6.0 -Normal Goat serum \(Vector Laboratories; S-1000) -Carrageenan Lambda \(Sigma-Aldrich, C-3889) -TritonX-100 \(Sigma-Aldrich, T8787) -OCT Compound $\backslash($ VWR, 361603E) -Vectashield Mounting Medium \(Vector Laboratories H-1000) -Anti- 
preproenkephalin rabbit antiserum \(Neuromics, RA14124) -Anti-rabbit IgG Alexa488-conjugated secondary antibody \(Invitrogen, A-11008) -DAPI \(Molecular Probes, D-1306)

\section{Equipment}

-Peristaltic Pump \(Masterflex L/S) -Cryostat \(Leica CM1950) -Rocker

\section{Procedure}

1. Anesthetize the mouse by intraperitoneal injection of sodium pentobarbital according to manufacturer guideline. 2. Immobilize the fully anesthetized animal on an appropriate surface surrounded by a plastic container for recovery of any biological fluid. 3. Cut open the chest and secure the tissue in order to obtain visibility of the hart. 4. Insert a 20-gauge needle hooked up to a perfusion pump into the left ventricle. 5. Perform a small incision into the left atrium and perfuse the animal at $3 \mathrm{ml} / \mathrm{min}$ for 3 minutes with TBS. 6. Switch the perfusion to $\mathrm{CS}$ at $3 \mathrm{ml} / \mathrm{min}$ for additional 3 minutes. 7. Remove the brain from the skull and postfix it in CS for $1 \mathrm{~h}$ at RT. 8. Wash the tissue twice with TBS to remove residual CS and place it in CPB until it sinks to the bottom of the tube. 9. Wash the brain twice with TBS to remove excess sucrose 10 . Embed the brain in OCT and snap-freeze it using liquid nitrogen. Store blocks at $-80^{\circ} \mathrm{C}$ until use. 11. Prepare cryosections of 30-to-40 $\mu \mathrm{m}$ thickness in the appropriate orientation using a cryostat. Place free-floating sections in 24 well plates with $300 \mu$ TBS maintaining the sequential order. 12 . Wash the sections twice with $300 \mu \mathrm{l}$ TBS for $10 \mathrm{~min}$. 13. Incubate for $30 \mathrm{~min}$ at RT with QS. 14 . Wash three times with $300 \mu \mathrm{l}$ WS. 15. Incubate for $1 \mathrm{~h}$ at RT with BB. 16. Remove BB and incubate tissues with $200 \mu \mathrm{l}$ AlB with 1:200 dilution of anti-preproenkephalin antibody and incubate overnight at $4^{\circ} \mathrm{C}$ with gentle rocking. 17. Wash tissues three times as above. 18. Incubate with Alexa488-cognugated secondary antibody 1:1000 dilutions in AIB for $3 \mathrm{~h}$ at RT with gentle rocking. 19. Wash three times as above 20. Counterstain the tissue with $100 \mathrm{ng} / \mathrm{ml}$ DAPI for 10 minutes at RT 21. Wash once as above 22. Place sections on a gelatin-coated slide using a fine brush 23 . As soon as the sections appear dry mount the slides using Vectashield Mounting Medium and cover with a coverslip glass 24. Seal the edges of the coverslip with nail polish to reduce oxidation of the mounting medium 25. Let the slides dry at RT for 30 minutes and then proceed with the analysis.

\section{Timing}

The staining procedure takes a total of $24 \mathrm{~h} \backslash$ (including an overnight incubation) but excluding the time needed to section the tissues at the cryostat.

\section{Troubleshooting}

For best results the staining should be performed within a week from the sectioning of the OCT block. Best is freshly sectioned. If prolonged exposure to crosslinking fixative cannot be avoided, an antigen retrieval step becomes necessary for a successful detection of the peptide. In this case staining can be 
improved by adding the following additional steps after point 11: 11a Pre-heat a cooker or water bath containing a heat-resistant holder for sections with SCB to $80^{\circ} \mathrm{C} \backslash$ (optimal temperature must be determined by the user and depends on the tissue thickness and extension of crosslinking time). 11b Immerse sections in the solution and incubate for 10 minutes $\backslash$ (optimal incubation time must be determined by the user and depends on the tissue thickness and extension of crosslinking time). 11c Turn off steamer or water bath and move the sections to room temperature $11 \mathrm{~d}$ Let sections cool at room temperature for 20 minutes. 11e Rinse the sections in WS twice for 10 minutes. Proceed as above from step 12.

\section{Anticipated Results}

A clear cytoplasmic/perinuclear staining is expected in the majority of striatal cells $\backslash$ (see Figure $1 \mathrm{~b}$ from Besusso et al. $\left.{ }^{(8)}\right)$.

\section{References}

1. Vonsattel, J.P., DiFiglia, M. Huntington Disease. J Neuropathol Exp Neurol. 57, 369-384 (1998). 2. Andrew, S.E. et al. The relationship between trinucleotide $\backslash(C A G)$ repeat length and clinical features of Huntington's disease. Nat Genet 4, 398-403 \(1993). 3. Richfield, E.K., Maguire-Zeiss, K.A., Cox, C., Gilmore, J., Voorn, P. Reduced expression of preproenkephalin in striatal neurons from Huntington's disease patients. Ann Neurol. 37, 335-343 \(1995). 4. Steiner, H., Gerfen, C.R. Role of dynorphin and enkephalin in the regulation of striatal output pathways and behavior. Exp Brain Res. 123, 60-76 \(1998). 5. Menalled, L. et al. Decrease in striatal enkephalin mRNA in mouse models of Huntington's disease. Exp Neurol. 162, 328-342 \(2000). 6. Docherty, K., Steiner, D.F. Post-translational proteolysis in polypeptide hormone biosynthesis. Annu Rev Physiol. 44, 625-638 \(1982). 7. Przewlocki, R., Przewlocka, B. Opioids in chronic pain. Eur J Pharmacol. 429, 79-91 \(2001). 8. Besusso, D. et al. BDNF-TrkB Signaling in Striatopallidal Neurons Controls Inhibition of Locomotor Behaviour. Nature Commun. 4 18/06/2013 | doi:10.1038/ncomms3031. 